\begin{tabular}{|c|c|c|}
\hline $\begin{array}{r}\text { PRAMANA } \\
- \text { journal of } \\
\text { physics }\end{array}$ & (c) Indian Academy of Sciences & $\begin{array}{l}\text { Vol. } 79, \text { No. } 3 \\
\text { September } 2012 \\
\text { pp. } 471-481\end{array}$ \\
\hline
\end{tabular}

\title{
Probing ultrafast carrier dynamics, nonlinear absorption and refraction in core-shell silicon nanowires
}

\author{
SUNIL KUMAR $^{1, *}$, M KHORASANINEJAD ${ }^{2}$, M M ADACHI $^{2}$, \\ K S KARIM ${ }^{2}$, S S SAINI ${ }^{2}$ and A K SOOD ${ }^{1}$ \\ ${ }^{1}$ Department of Physics and Centre for Ultrafast Laser Applications, Indian Institute of Science, \\ Bangalore 560 012, India \\ ${ }^{2}$ Department of Electrical and Computer Engineering, University of Waterloo, Canada N2L 3G1 \\ *Corresponding author. E-mail: skumar@physics.iisc.ernet.in
}

MS received 9 March 2012; accepted 18 April 2012

\begin{abstract}
We investigate the relaxation dynamics of photogenerated carriers in silicon nanowires consisting of a crystalline core and a surrounding amorphous shell, using femtosecond timeresolved differential reflectivity and transmission spectroscopy at $3.15 \mathrm{eV}$ and $1.57 \mathrm{eV}$ photon energies. The complex behaviour of the differential transmission and reflectivity transients is the mixed contributions from the crystalline core and the amorphous silicon on the nanowire surface and the substrate where competing effects of state-filling and photoinduced absorption govern the carrier dynamics. Faster relaxation rates are observed on increasing the photogenerated carrier density. Independent experimental results on crystalline silicon-on-sapphire (SOS) help us in separating the contributions from the carrier dynamics in crystalline core and the amorphous regions in the nanowire samples. Further, single-beam z-scan nonlinear transmission experiments at $1.57 \mathrm{eV}$ in both open- and close-aperture configurations yield two-photon absorption coefficient $\beta(\sim 3 \mathrm{~cm} / \mathrm{GW})$ and nonlinear refraction coefficient $\gamma\left(-2.5 \times 10^{-4} \mathrm{~cm}^{2} / \mathrm{GW}\right)$.
\end{abstract}

Keywords. Silicon; nanowires; femtosecond; ultrafast spectroscopy; nonlinear optical coefficients.

PACS Nos 78.40.Fy; 78.67.Uh; 78.47.J-

\section{Introduction}

Energy relaxation processes play an important role in hot carrier transport in semiconductor-based fast electronic and optoelectronic devices. Spatial confinement of charge carriers in semiconducting nanostructures not only leads to band-gap enhancement [1] but also gives rise to surface-induced charge states in the forbidden gap. Silicon nanowires are being used to fabricate multidevices such as transistors, photoemitters and photosensors, all on a single chip [2-4], mainly due to the ease of large-scale fabrication and integration [5]. Crystalline core-amorphous shell silicon heterostructures have 


\section{Sunil Kumar et al}

been recently used in applications including battery electrodes [6], electrical switching [7] and solar cells [8]. The advantage of using core-shell nanowires over purely crystalline or amorphous silicon nanowires is the passivation of crystalline silicon to be used as an efficient electrical conducting pathway [6] together with high optical absorption by amorphous silicon. This makes them suitable for solar cell applications [9]. Ultrafast time-resolved pump-probe spectroscopy has helped us in understanding the energy relaxation processes in various semiconducting nanowires [10-13]. Recently, pump-probe spectroscopy was reported on randomly networked crystalline silicon nanowires (SiNWs) on a sapphire substrate where the electron relaxation rate was found to be dependent on the nanowire diameter and the energy of the probed states within the forbidden energy gap [14]. Inspired by these studies, we have taken up the present study on crystalline core-amorphous shell silicon nanowires for finding out the carrier relaxation dynamics and nonlinear optical properties as these nanowires have potential applications in silicon-based visible light modulators and all-optical switching devices [15].

We performed femtosecond optical measurements using 70 fs laser pulses, on preferentially vertically grown crystalline core-amorphous shell silicon nanowires on a glass substrate. Electron-hole pairs are optically injected into the nanowires and the subsequent temporal evolution of their population distributions are studied with femtosecond time resolution in the pump-probe reflection and transmission experiments. We examine the dependence of the photogenerated carrier dynamics on the pump-photon energy $(3.15 \mathrm{eV}$ and $1.57 \mathrm{eV})$ and the pump fluence. The transient differential reflectivity $(\Delta R / R)$ and transmission $(\Delta T / T)$ signals have contributions from both the crystalline core and the amorphous silicon shell on the nanowire surface, leading to a complex behaviour of the transients that reveal multiple relaxation components of the carrier dynamics with time constants ranging from sub-ps to many hundreds of ps governed by the photoinduced absorption and the state-filling effects. Independent experiments performed under the same experimental conditions on crystalline silicon-on-sapphire (SOS), on the other hand, reveal only single exponential decay of the photoinduced transmission transients with time constant of $\sim 500$ ps. Also, we have estimated two-photon absorption coefficient $\beta \sim 3 \mathrm{~cm} / \mathrm{GW}$ and negative nonlinear refractive index $\gamma \sim-2.5 \times$ $10^{-4} \mathrm{~cm}^{2} / \mathrm{GW}$ from z-scan experiments on the nanowires at $790 \mathrm{~nm}$.

\section{Experimental}

The core-shell silicon nanowires were fabricated by the vapour-liquid-solid method in a plasma-enhanced chemical-vapour-deposition system on Corning 1737 glass substrate [9]. A $2 \mathrm{~nm}$ thick layer of $\mathrm{Sn}$ was deposited by e-beam evaporation followed by annealing at $400^{\circ} \mathrm{C}$ to form $\mathrm{Sn}$ droplets used as the catalyst for nanowire growth. Silane was used as the source gas and nanowire growth temperature was $400^{\circ} \mathrm{C}$. High resolution electron microscopy showed that the crystalline core has a diameter of about $10 \mathrm{~nm}$ which is surrounded by an amorphous silicon shell [9] resulting in a total nanowire average diameter of $\sim 60 \mathrm{~nm}$ as seen from the scanning electron microscopic topograpy in figure 1a. Raman spectrum of the nanowire sample shows a sharp peak at $520 \mathrm{~cm}^{-1}$ due to the highly crystalline core and a broad peak at $480 \mathrm{~cm}^{-1}$ due to the scattering from amorphous silicon 

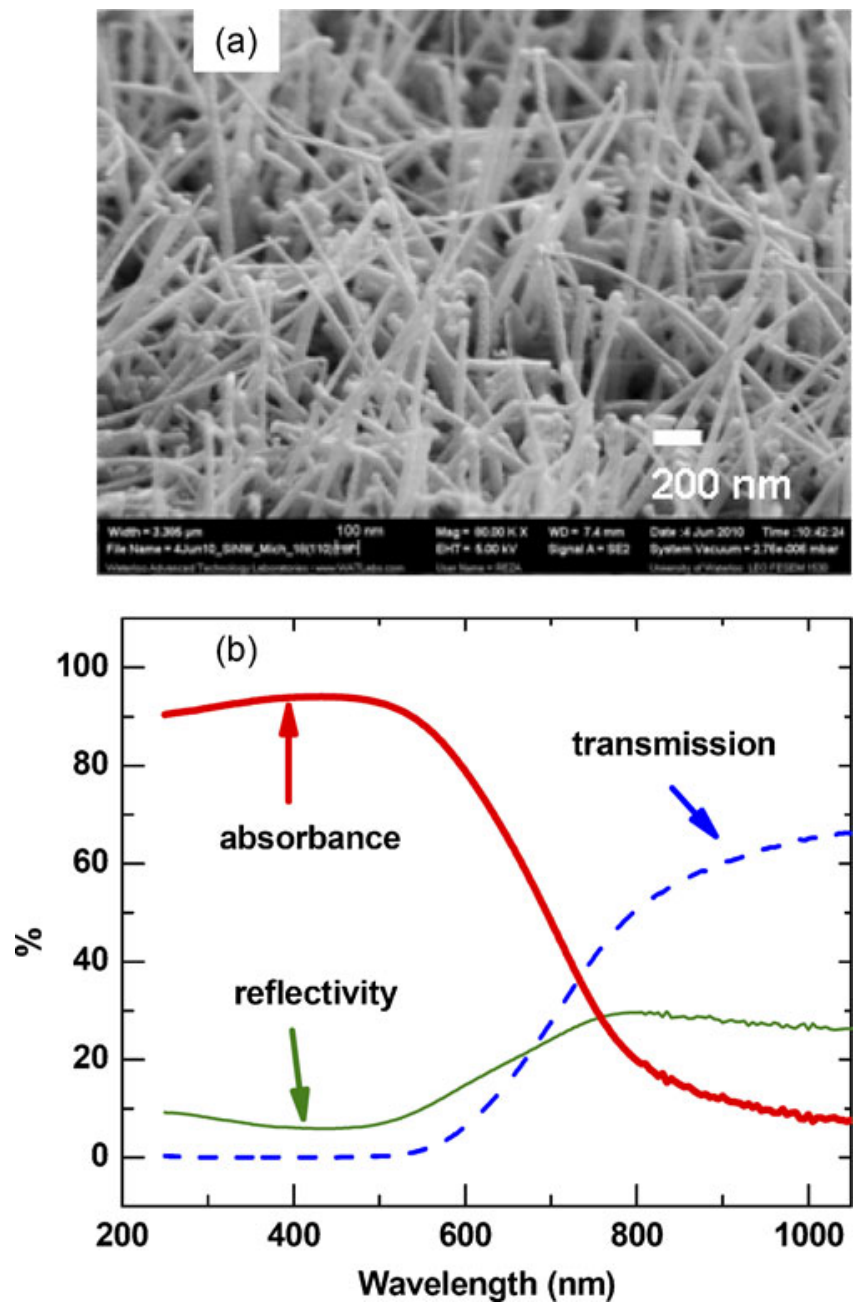

Figure 1. Characterization of the silicon nanowires deposited on Corning 1767 glass [15]. (a) Scanning electron microscopic topography for estimating the average total diameter of about $60 \mathrm{~nm}$ of the core-shell silicon nanowires, (b) linear optical absorption measurement using a visible-infrared absorption spectrometer.

on the nanowires and on the substrate [9]. The overall thickness of the silicon nanowire film was $\sim 600 \mathrm{~nm}$ measured independently using atomic force microscopy providing an estimate of the nanowire length.

The linear optical reflectivity and transmission data close to normal incidence obtained using the conventional visible-IR spectrophotometer are shown in figure $1 \mathrm{~b}$ along with the calculated absorbance. The band gap of the amorphous silicon (a-Si) is around $1.78 \mathrm{eV}$ $(\sim 700 \mathrm{~nm}$ ) whereas the absorption from the nanowire sample is extended much beyond this wavelength and can originate mostly from the crystalline silicon core [9]. The linear 
absorbance at $395 \mathrm{~nm}$ is about $95 \%$ which corresponds to the absorption coefficient $\alpha \sim$ $4.5 \times 10^{4} \mathrm{~cm}^{-1}, \sim 14$ times higher than at $790 \mathrm{~nm}$.

Femtosecond laser pulses centred at $790 \mathrm{~nm}(1.57 \mathrm{eV})$ from a Ti:sapphire regenerative amplifier providing laser pulses at $1 \mathrm{kHz}$ repetition rate and at $395 \mathrm{~nm}(3.15 \mathrm{eV})$ generated in a beta barium borate crystal, were used in the present degenerate and nondegenerate pump-probe experiments in a noncollinear geometry. The laser beam size on the sample was larger than $1 \mathrm{~mm}$ which results in averaging over a large number of nanowires. For pump fluence-dependent measurements we used neutral density filters in the pump beam path. We examined the dependence of pump-photon energy $(1.57 \mathrm{eV}$ and $3.15 \mathrm{eV})$ and pump fluence on the carrier dynamics by measuring the time-resolved photoinduced changes in sample transmission $(\Delta T)$ and reflection $(\Delta R)$ normalized with respect to their normal values $\left(T\right.$ and $R$ ) at $1.57 \mathrm{eV}$ probe with a probe fluence of $\sim 45 \mu \mathrm{J} / \mathrm{cm}^{2}$. All measurements were performed at room temperature with pump and probe polarizations crossed with each other and an analyser crossed with the pump polarization was placed at the detector. For the nonlinear transmission single-beam z-scan measurements at $790 \mathrm{~nm}$, we used a focussing lens (focal length $=15 \mathrm{~cm}$ ) to continuously vary the laser power on the sample from $2 \mathrm{MW} / \mathrm{cm}^{2}$ to $\sim 30 \mathrm{GW} / \mathrm{cm}^{2}$.

In our experiments, the probe pulse centred at $1.57 \mathrm{eV}$ is in the band-gap region of a-Si where there are no optically active energy levels as suggested from the strong suppression of optical absorption beyond $1.78 \mathrm{eV}$ [9]. A pump-probe signal at this probe energy thus signifies a finite absorption at $1.57 \mathrm{eV}$ mostly due to phonon-assisted indirect transitions in the crystalline silicon (c-Si) core and possibly a contribution from the band-tail states in a-Si. Moreover, the diameter of the crystalline core is $\sim 10 \mathrm{~nm}$ where quantization effects are weak.

\section{Results and discussion}

Differential transmission transients $(\Delta T / T)$ from the SiNWs at $3.15 \mathrm{eV}$ pump collected at various pump fluences are presented in figure $2 \mathrm{a}$. The recovery of the signals has three components with negative and positive amplitudes and time constants from sub-ps to ns arising from competing photoinduced absorption and state-filling effects. A sum of exponentially decaying functions, $\sum_{k}\left(1-\mathrm{e}^{-t / \tau_{\mathrm{r}}}\right) A_{k} \mathrm{e}^{-t / \tau_{k}}$, has been used to capture the complete dynamics of the photoinjected carriers in the SiNWs. Here the first term inside the brackets is used to capture the initial rise time $\left(\tau_{\mathrm{r}}\right)$ of the signal and corresponds to the thermalization of the photocarriers by carrier-carrier and/or carrier-optical phonon scattering, and $A_{k}$ and $\tau_{k}$ are the amplitudes and decay times of various components in the signal as displayed in figure $2 \mathrm{~b}$. The fits are shown by thick continuous lines in figure $2 \mathrm{a}$. We clearly see two negative components, one very fast with relaxation time $\tau_{1} \sim 1.6 \mathrm{ps}$ and another very slow with $\tau_{3}>1$ ns attributed to the photoinduced absorption of carriers whereas at intermediate times we have a positive component with relaxation time constant $\tau_{2} \sim 90 \mathrm{ps}$ that can arise from state-filling effects-induced saturation of carrier population in the energy states at the probe-photon energy, thus blocking the normal absorption of the probe photons in transition from the valence band to the conduction band. The long-lived negative component could be a result of photoinduced absorption of the probe photons from a set of low-energy defect states to the conduction band before the carrier 

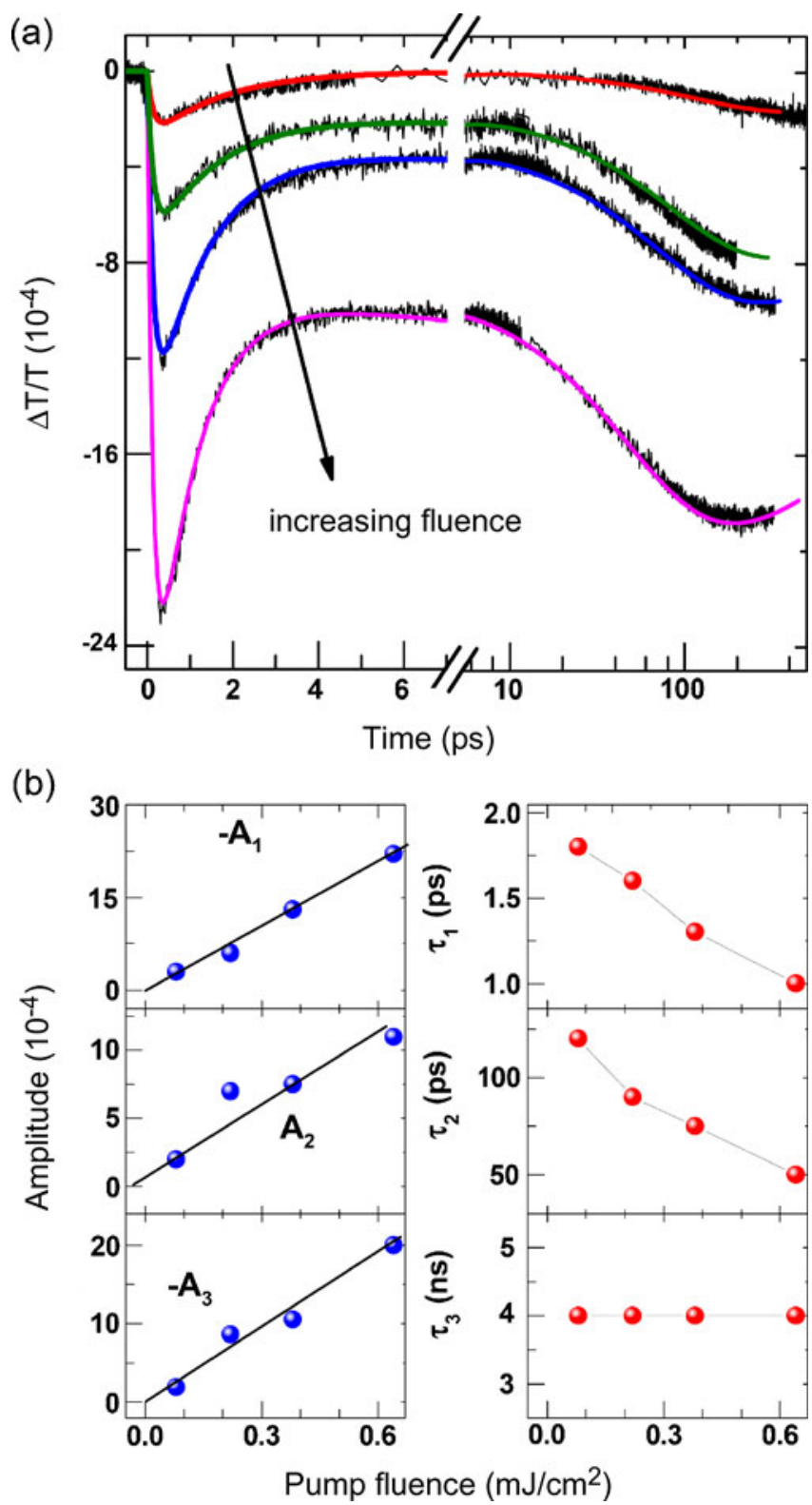

Figure 2. Differential transmission transients from the SiNW sample measured at various pump fluences with pump excitation at $3.15 \mathrm{eV}$. (a) The raw data with logarithmic $x$-axis at longer times are shown for clarity and (b) the fluence dependence of amplitude $(A)$ and time constant $(\tau)$ of three exponentially decaying relaxation components of the signals. 


\section{Sunil Kumar et al}

recombination takes place to reach a complete equilibrium. Radiative recombination lifetimes varying from picoseconds in defect-free states to tens of nanoseconds in defect states in SiNWs have been suggested from time-resolved photoluminescence studies [16].

The pump-fluence dependence of each component is shown in figure $2 b$ where a linear increase in the amplitudes and faster relaxation times (except $\tau_{3}$ ) can be seen at higher fluences. The rise time $\tau_{\mathrm{r}} \sim 150 \mathrm{fs}$ is found to be fluence-independent. In nanowires, the carrier-carrier interactions are larger due to the spatial confinement of carriers when compared to bulk semiconductor, leading to dominant Augur recombination process in the relaxation [14] according to which the relaxation time decreases with increasing fluence. Also, at high fluences, the photogenerated carrier density becomes sufficiently high to cause band-gap renormalization [14] and hence pronounced effects in the carrier dynamics at high fluences. Generally, Auger recombination mechanism is suggested to operate at carrier densities larger than $10^{21} \mathrm{~cm}^{-3}$ [17]. However, the maximum pump fluence at $395 \mathrm{~nm}$ that is used in our experiments $\left(620 \mu \mathrm{J} / \mathrm{cm}^{2}\right)$, corresponds to a photogenerated free carrier density $N \sim 5.4 \times 10^{19} \mathrm{~cm}^{-3}$ where Auger recombination mechanism is not expected for the carrier relaxation. Though the decrease in the relaxation times $\tau_{1}$ and $\tau_{2}$ is observed at $395 \mathrm{~nm}$ pump, the same is found to be absent when $790 \mathrm{~nm}$ pump was used (discussed later). Moreover, in a-Si, the carrier relaxation time as fast as 0.6 ps was reported to be fluence-independent even at carrier densities much higher than ours [17]. On the other hand, in crystalline semiconducting nanowires, fluence-dependent relaxation times have been observed where Auger process plays a role [10,14]. These observations may suggest that the origin of $\tau_{1}$ and $\tau_{2}$ is not simply related to the carrier relaxation either in a-Si or in $\mathrm{c}-\mathrm{Si}$ in the nanowire core separately; rather the presence of one affects the other.

We believe that in the present heterostructure SiNWs, both the crystalline core and the amorphous silicon on the nanowire surface contribute to the observed decay of the signals. The optical penetration depth $(\sim 1 / \alpha)$ at $395 \mathrm{~nm}$ is about $220 \mathrm{~nm}$ and much larger $(\sim 3 \mu \mathrm{m})$ at $790 \mathrm{~nm}$ which effectively means that the photon absorption (at both $395 \mathrm{~nm}$ and $790 \mathrm{~nm}$ ) takes place not only in the amorphous surface regions of the nanowires but also inside the crystalline core creating electron-hole pairs. Therefore, both the c- and a-Si are contributing to the measured transients. We may emphasize that much of the incident light is transmitted to the substrate but the glass or the sapphire alone does not show any pump-probe signal [18]. Very slow $(\sim \mathrm{ns})$ relaxation time in crystalline bulk silicon is commonly known $[19,20]$ whereas much faster relaxation times from tens of ps to sub-ps have been reported for a-Si $[17,18,21]$. To decipher the role of the crystalline core and that of the a-silicon, we carried out experiments under the same experimental conditions on commercially available crystalline silicon-on-sapphire (SOS) (0.4 $\mu \mathrm{m} \mathrm{c-Si}$ film on $0.5 \mathrm{~mm}$ sapphire) at $395 \mathrm{~nm}$ and $790 \mathrm{~nm}$ pump. The results are displayed in figure 3. In this case we observe a single exponential decay of the photocarriers with relaxation time constant $\tau \sim 500 \mathrm{ps}$, independent of the excitation wavelength and the pump fluence or the photogenerated carrier density. The only difference in the carrier dynamics at the two pump wavelengths is in their population build up immediately after the pump excitation (inset of figure 3). At $395 \mathrm{~nm}$, the carriers are injected into states in the conduction band far off from the Fermi energy and hence take more time $\left(\tau_{\mathrm{r}} \sim 0.9 \mathrm{ps}\right)$ to reach the states connected by the probe $(790 \mathrm{~nm})$ than they take in the case of excitation with the $790 \mathrm{~nm}$ pump $\left(\tau_{\mathrm{r}} \sim 0.4 \mathrm{ps}\right)$. 


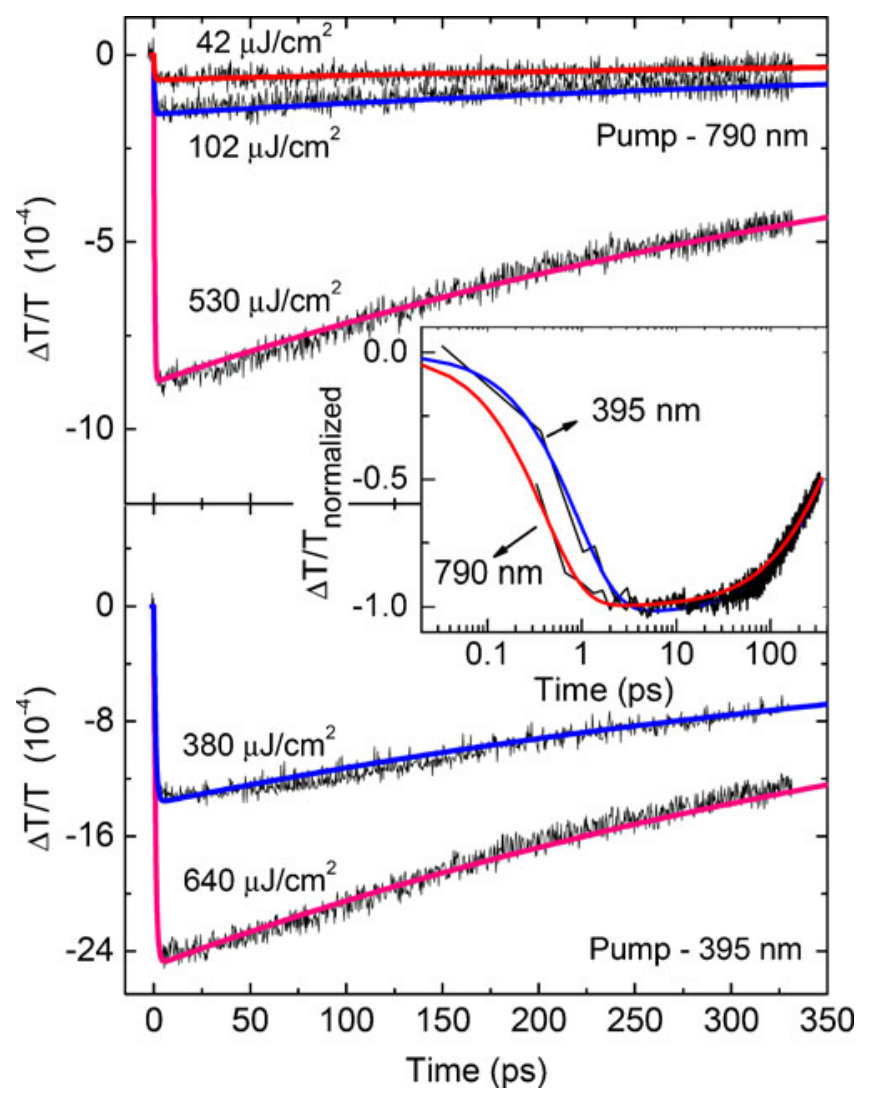

Figure 3. Differential transmission transients from SOS measured using pump excitation at $790 \mathrm{~nm}$ (top panel) and $395 \mathrm{~nm}$ (bottom panel) at various pump fluences. The continuous lines are fits using a rising contribution and a single exponential decay. The inset displays the semi-logarithmic plot to clearly show the difference in the rising time of the signals at the two pump wavelengths.

From the above discussion, the initial fast negative component with decay time constant $\tau_{1} \sim 1.6 \mathrm{ps}$ can be attributed to the photoinduced absorption where the pump-injected carriers in the excited states undergo secondary excitation by the probe photons and free carriers are contributed in the amorphous regions (band-gap energy $=1.78 \mathrm{eV}$ ). In c-Si, the lowest band-gap (indirect) energy is $1.12 \mathrm{eV}$ and the band gap energy for direct transition is much larger than the single photon energies used in our experiments. Amorphous silicon, on the other hand, with a direct band-gap energy of $1.78 \mathrm{eV}$ [9] is highly absorbing at photon energies above $1.78 \mathrm{eV}$ because the momentum is not conserved during interband transitions due to atomic disorder [22]. Also, electrons and holes by dangling bond defects occur in a-Si [23]. Therefore, a pump-probe signal at a probe photon energy of $1.57 \mathrm{eV}$ which is in the mid-gap region for both the c- and a-Si effectively means that the sample has a finite absorption at $790 \mathrm{~nm}$ which gets affected by the pump excitation. The probe can be absorbed through indirect (phonon-assisted) transition in the c-Si and 
electronic transitions to the band-tail states in the a-Si. The slow relaxation components ( $\tau_{2} \sim 100 \mathrm{ps}$ and $\tau_{3}>1 \mathrm{~ns}$ ) are due to the relaxation of the photogenerated carriers in the crystalline core region of the nanowires. The slower one $\left(\tau_{3}\right)$ is nearly fluenceindependent while $\tau_{2}$ is fluence-dependent (figure $2 \mathrm{~b}$ ). The latter can be related to the carrier trapping in the crystalline-amorphous interface of the core-shell nanowires.

Interestingly, when the pump-probe signal was collected in the reflection geometry $(\Delta R / R)$, we observed a clear difference in the signal behaviour at faster times (below $5 \mathrm{ps}$ ). Figure 4 displays the results of this comparison between the $\Delta R / R$ and $\Delta T / T$ signals measured using at $395 \mathrm{~nm}$ pump at a pump fluence of $\sim 220 \mu \mathrm{J} / \mathrm{cm}^{2}$. The initial decrease in both the signals just after photoexcitation takes about $100 \mathrm{fs}$ in $\Delta R / R$ and $\sim 150 \mathrm{fs}$ in $\Delta T / T$ to reach the minimum. At longer times, larger than $\sim 20 \mathrm{ps}$, both the signals $(\Delta R / R$ and $\Delta T / T)$ behave the same way. However, their behaviour will be different at shorter times where a single negative component in $\Delta T / T$ but one negative and one positive component in the $\Delta R / R$ signal are visible. It appears that the fast negative component in $\Delta T / T$ has two contributions, one from the a-Si on the nanowire and the
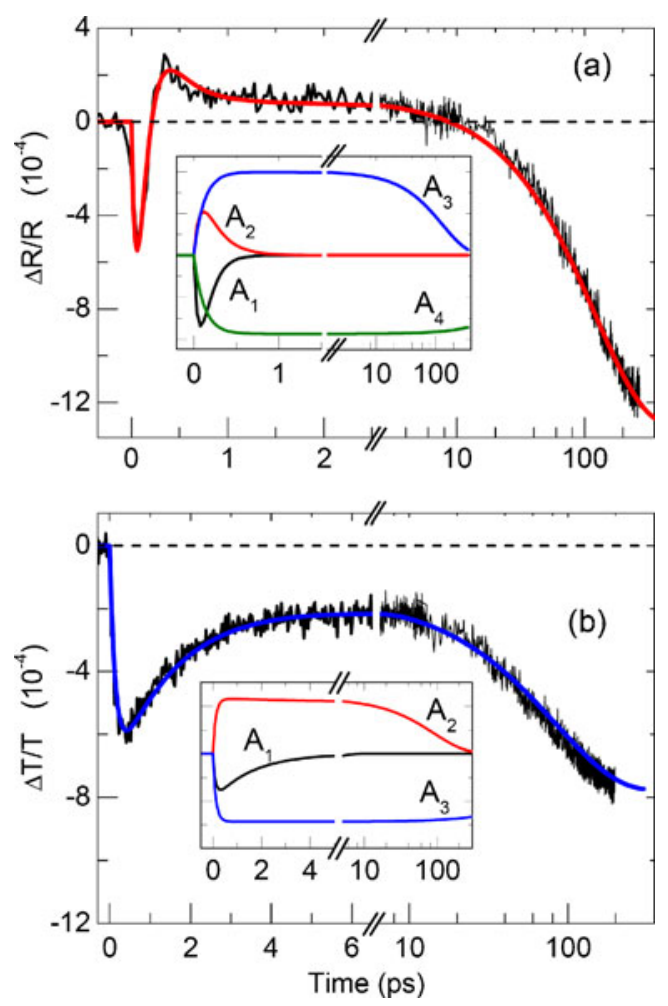

Figure 4. Differential reflectivity and transmission transients from core-shell SiNWs using pump excitation at $3.15 \mathrm{eV}$ with fits shown by thick continuous lines, using a sum of exponentially decaying functions. The insets show four (three) individual components used in fitting the $\Delta R / R(\Delta T / T)$ signal. Note that for clarity of presentation, the $x$-axis is linear at short times and logarithmic after the break. 
other from that on the substrate. These get separated out in the $\Delta R / R$ geometry due to the change in polarity of the signal-component contributed by the residual a-Si on the substrate.

The pump-probe signal immediately after the pump excitation is a consequence of cascaded absorption of a pump photon and a probe photon in nonresonant experiments like ours. In that case the magnitude of the signal should be smaller at $790 \mathrm{~nm}$ pump when compared with that at $395 \mathrm{~nm}$ pump because of very small density of states near the band tails of a-Si band gap $(700 \mathrm{~nm})$. This is indeed the case as we have seen for the SOS (figure 3). To verify this point further, we pursued degenerate pump-probe measurements at $790 \mathrm{~nm}$ on the SiNWs as well. The results are displayed in figure 5. We again see the initial photoinduced absorption with a rise time of $\tau_{\mathrm{r}} \sim 120 \mathrm{fs}$, nearly identical to that at $395 \mathrm{~nm}$ pump. As can be seen, the magnitude of the signal near the zero delay is much smaller when compared to the $395 \mathrm{~nm}$ pump excitation (figure 2) at the same pump fluence. This is expected due to much smaller absorption in the crystalline core as well as the band tails of the a-Si at $790 \mathrm{~nm}$. Here, the combined absorption of one pump and one probe photon is responsible for the sharp dip at the zero delay. A positive component with a decay time of $\sim 10$ ps could be identified only at the highest experimental pumpfluence (figure 5) and the data at all the fluences could be fitted using only two negative components with decay times of $\tau_{1} \sim 200 \mathrm{fs}$ and $\tau_{2} \sim 4 \mathrm{~ns}$. The relaxation times are found roughly fluence-independent unlike the $395 \mathrm{~nm}$ pump as discussed before and the linear dependence of the amplitudes $A_{1}$ and $A_{2}$ can be seen in the inset of figure 5 .

The photoinduced absorption near the zero delay in the degenerate pump-probe signal at $790 \mathrm{~nm}$ (figure 5) can be used to estimate the two-photon absorption coefficient $\beta$ of

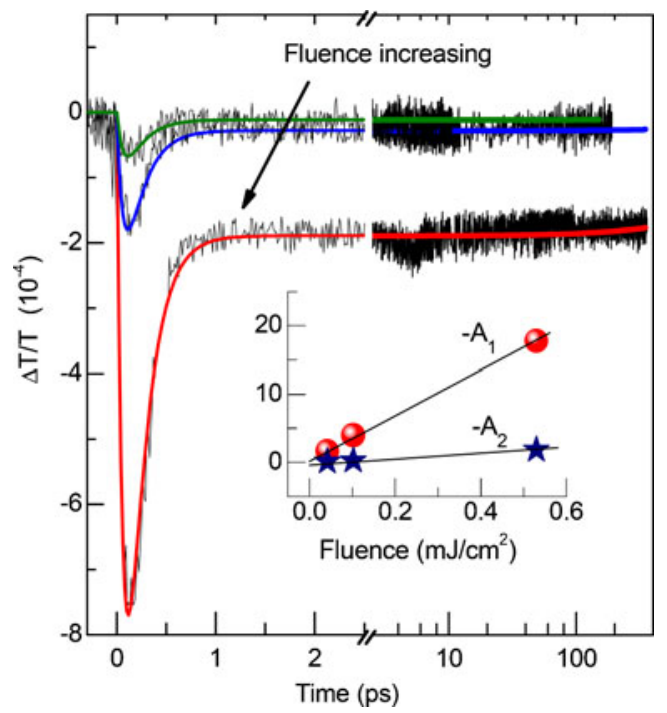

Figure 5. Differential transmission transients for SiNWs using $790 \mathrm{~nm}$ pump at various pump fluences. The inset shows the linear fluence dependence of the magnitudes of two individual relaxation components in the signal but the time-constants remain fluence-independent. 


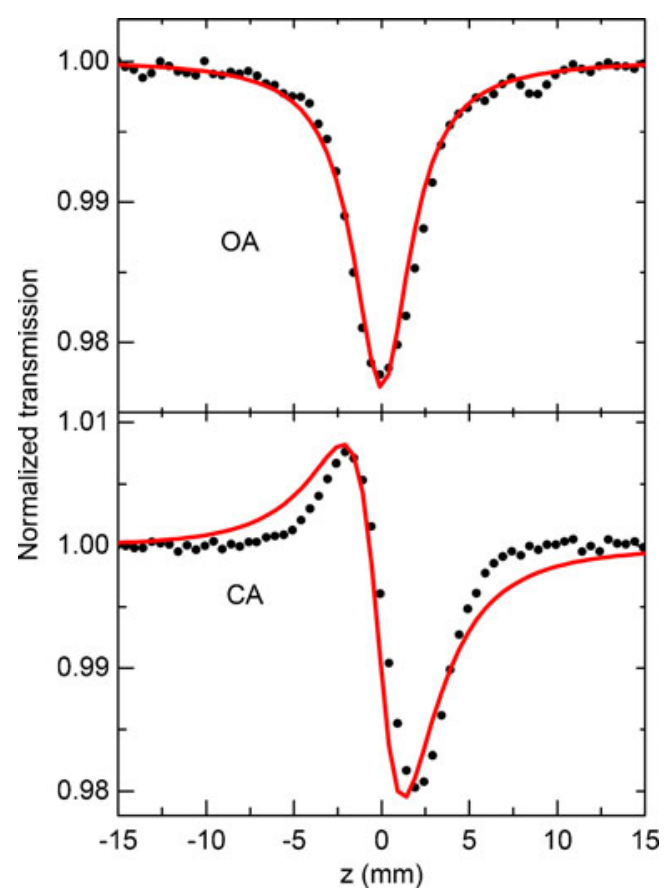

Figure 6. Open-aperture and close-aperture z-scan results from silicon nanowire film at $790 \mathrm{~nm}$. The continuous lines are theoretical fits to estimate the two-photon absorption coefficient and nonlinear refractive index.

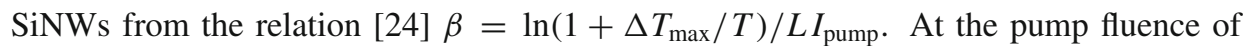
$530 \mu \mathrm{J} / \mathrm{cm}^{2}, \beta$ is $\sim 1.3 \mathrm{~cm} / \mathrm{GW}$. This is compared with the results obtained from openaperture (OA) and close-aperture (CA) z-scan measurements. Optical limiting behaviour arising from two-photon absorption at $790 \mathrm{~nm}$ in the OA and negative refractive nonlinearity $(\gamma)$ in the CA z-scan results can be seen in figure 6. Consistent theoretical fits [25] to the OA and CA data using $\alpha(I)=\alpha_{0}+\beta I, n(I)=n_{0}+\gamma I$ where $\alpha_{0}$ and $n_{0}$ are the linear absorption and refraction coefficients, give $\beta \sim 3 \mathrm{~cm} / \mathrm{GW}$ and $\gamma \sim-2.5 \times 10^{-4} \mathrm{~cm}^{2} / \mathrm{GW}$. The value of $\gamma$ is small but $\beta$ is significant when compared with highly nonlinear materials such as graphene and $\mathrm{BCN}[24,25]$ in the visible range. Similar value of $\beta(\sim 4 \mathrm{~cm} / \mathrm{GW})$ and a smaller but negative nonlinear refractive index was reported previously for a-Si waveguides [21] which are much higher than those for the c-Si [26].

\section{Conclusions}

To summarize, we have studied the photoinduced carrier dynamics in core-shell silicon nanowires where we observe the combination of fast carrier relaxation within $1.6 \mathrm{ps}$ at $395 \mathrm{~nm}$ pump and $0.2 \mathrm{ps}$ at $790 \mathrm{~nm}$ pump in the amorphous surface, and very slow relaxation larger than $100 \mathrm{ps}$ in the crystalline core region of the nanowires. The large 
values of optical nonlinearities estimated from z-scan measurements along with the ultrafast response of better than 1.6 ps mainly in the a-Si shell make the core-shell SiNWs a good candidate for potential applications in silicon-based visible light modulators and all-optical-switching devices.

\section{Acknowledgements}

AKS and SK acknowledge the financial support from the Department of Science and Technology, India.

\section{References}

[1] D D D Ma, C S Lee, F C K Au, S Y Tong and S T Lee, Science 299, 1874 (2003)

[2] Y Cui, Z Zhong, D Wang, W U Wang and C M Lieber, Nano Lett. 3, 149 (2003)

[3] P Servati, A Colli, S Hofmann, Y Q Fu, P Beecher, Z A K Durrani, A C Ferrari, A J Flewitt, J Robertson and W I Milne, Physica E38, 64 (2007)

[4] R Agarwal and C M Lieber, Appl. Phys. A85, 209 (2006)

[5] S T Picraux, S A Dayeh, P Manandhar, D E Perea and S G Choi, J. Mater. 62, 35 (2010)

[6] L F Cui, R Ruffo, C K Chan, H Peng and Y Chui, Nano Lett. 9, 491 (2008)

[7] Y Dong, G Yu, M C McAlpine, W Lu and C M Lieber, Nano Lett. 8, 386 (2008)

[8] M Taguchi, K Kawamoto, S Tsuge, T Baba, H Sakata, M Morizane, K Uchihashi, N Nakamura, S Kiyama and O Oota, Prog. Photovoltaics 8, 503 (2000)

[9] M M Adachi, M P Anantram and K S Karim, Nano Lett. 10, 4093 (2010)

[10] R P Prasankumar, S Choi, S A Trugman, S T Picraux and A J Taylor, Nano Lett. 8, 1619 (2008)

[11] C-K Sun, S-Z Sun, K-H Lin, K Y-J Zhang, H-L Liu, S-C Liu and J-J Wu, Appl. Phys. Lett. 87, 023106 (2005)

[12] A Othonosa, E Lioudakis, U Philipose and H E Ruda, Appl. Phys. Lett. 91, 241113 (2007)

[13] C R Carey, Y Yu, M Kuno and G V Hartland, J. Phys. Chem. C113, 19077 (2009)

[14] A Kar, P C Upadhya, S A Dayeh, S T Picraux, A J Taylor and R P Prasankumar, IEEE J. Sel. Top. Quant. Electron. 17, 889 (2011)

[15] V R Almeida, C A Barrios, R R Panepucci and M Lipson, Nature 43, 1081 (2004)

[16] J Qi, A M Belcher and J M White, Appl. Phys. Lett. 82, 2616 (2003)

[17] K P H Lui and F A Hegmann, J. Appl. Phys. 93, 9012 (2003)

[18] K P H Lui and F A Hegmann, Appl. Phys. Lett. 78, 3478 (2001)

[19] K Narayana and S F Preble, Opt. Exp. 18, 8998 (2010)

[20] J Kudrna, P Maly, F Trojanek, J Stepanek, T Lechner, I Pelant, J Meier and U Kroll, Mater. Sci. Eng. B69-70, 238 (2000)

[21] K Ikeda, Y Shen and Y Fainman, Opt. Exp. 15, 17761 (2007)

[22] R A Street, Appl. Phys. Lett. 41, 1060 (1982)

[23] M M Adachi, M P Anantram and K S Karim, Nano Lett. 10, 4093 (2010)

[24] S Kumar, M Anija, N Kamaraju, K S Vasu, K S Subrahmanyam, A K Sood and C N R Rao, Appl. Phys. Lett. 95, 191911 (2009)

[25] S Kumar, N Kamaraju, K S Vasu, A Nag, A K Sood and C N R Rao, Chem. Phys. Lett. 499, $152(2010)$

[26] M Dinu, F Quochi and H Garcia, Appl. Phys. Lett. 82, 2954 (2003) 